\title{
Key Questions Relating to Left Ventricular Noncompaction Cardiomyopathy: Is the Emperor Still Wearing Any Clothes?
}

Anderson, RH; Jensen, B; Mohun, TJ; Petersen, SE; Aung, N; Zemrak, F; Planken, RN; Maclver, DH

For additional information about this publication click this link.

http://qmro.qmul.ac.uk/xmlui

Information about this research object was correct at the time of download; we occasionally make corrections to records, please therefore check the published record when citing. For more information contact scholarlycommunications@qmul.ac.uk 
Title page:

\section{Key Questions relating to left ventricular noncompaction cardiomyopathy - Is the Emperor still wearing any clothes?}

\section{Authors:}

Robert H Anderson ${ }^{1,2}$, Bjarke Jensen ${ }^{3}$, Timothy J Mohun ${ }^{4}$, Steffen E. Petersen ${ }^{5}$, Nay Aung ${ }^{5}$, Filip Zemrak, ${ }^{5}$ R Nils Planken, ${ }^{6}$ David H Maclver ${ }^{7,8,9}$

\section{Author affiliations}

1. Institute of Genetic Medicine, Newcastle University, Newcastle Upon Tyne, UK.

Email: sejjran@ucl.ac.uk

2. Division of Biomedical Sciences, University College London, London, UK.

3. Department of Anatomy, Embryology \& Physiology, Academic Medical Center, University of Amsterdam, The Netherlands. Email: b.jensen@amc.uva.nl

4. National Institute for Medical Research, London, UK. Email: tmohun@nimr.mrc.ac.uk

5. Barts Heart Centre, William Harvey Research Institute, NIHR Cardiovascular Biomedical Research Unit at Barts, Queen Mary University of London, London, United Kingdom. Email:

s.e.petersen@qmul.ac.uk/n.aung@qmul.ac.uk/f.zemrak@qmul.ac.uk

6. Department of Radiology, Academic Medical Center, University of Amsterdam, The Netherlands. Email: r.n.planken@amc.uva.nl

7. Department of Cardiology, Taunton \& Somerset Hospital, Musgrove Park, Taunton, TA1 5DA, UK Email: david.maciver@tst.nhs.uk

8. Biological Physics Group, School of Physics \& Astronomy, University of Manchester, Manchester, UK. 
9. University of Bristol, Senate House, Tyndall Avenue, Bristol BS8 1TH, UK.

Corresponding author: Prof. David H Maclver,

Running title: Noncompaction cardiomyopathy

Word count Main text: 4167. References: 66. Figures: 7.

Abbreviation used: ETrEF, excessive trabeculations with reduced ejection fraction. ETpEF, excessive trabeculations with preserved ejection fraction.

Funding Nil.

Acknowledgments None. We can confirm that there was no funding for this study

Competing interests: We confirm that we have no conflicts of interest.

\section{Contributors:}

RHA wrote the first draft and was responsible for the concept. TJM produced the episcopic datasets providing evidence regarding the fate of the trabecular component of the embryonic ventricular myocardium. BJ provided the developmental, comparative anatomy and embryological background. SEP, NA, and FZ provided clinical input, along with details of their investigations of the MESA cohort. RNP provided access to the Amsterdam cohort of patients diagnosed with "noncompaction cardiomyopathy". DHM provided input from his personal experience with patients having both excessive trabeculation and cardiomyopathy, and was responsible for the final editing. All authors provided critical revision of the manuscript and take responsibility for the contents, accuracy and integrity of the document. 


\title{
Key Questions relating to left ventricular noncompaction cardiomyopathy - Is the Emperor still wearing any clothes?
}

\begin{abstract}
The evidence is increasing that left ventricular noncompaction cardiomyopathy, as it is currently defined, does not represent failure of compaction of pre-existing trabecular myocardium found during embryonic development so as to form the compact component of the ventricular walls. Nor is there evidence, of which we are aware, to favour the notion that the entity is a return to a phenotype as seen in cold-blooded animals. It is also known that, when seen in adults, the presence of excessive ventricular trabeculations does not portend a poor prognosis when ejection fraction is normal, with the risks of complications such as arrhythmia and stroke being rare in this setting. It is also the case that images of "noncompaction" as provided from children, or in autopsy studies, are quite different from the features observed clinically in the asymptomatic adults with excessive trabeculation. Our review suggests that the presence of an excessively trabeculated left ventricular wall is not, in itself, a clinical entity. It is equally possible that the excessive trabeculation is no more than a bystander in the presence of additional lesions, such as dilated cardiomyopathy, with the additional lesions being responsible for the reduced ejection fraction bringing a given patient to clinical attention. We, therefore, argue that the term "noncompaction cardiomyopathy" is misleading, as there is neither failure of compaction nor a cardiomyopathic process in most individuals that fulfil widely used diagnostic criteria.
\end{abstract}

\section{$\underline{\text { Introduction }}$}

Although yet to be generally accepted, it is increasingly frequent to find the term "noncompaction cardiomyopathy" or "left ventricular noncompaction" used when excessive trabeculations are present in the left ventricle. As we will seek to establish in our review, there is no convincing evidence to show the finding of excessive trabeculation is representative of a discrete cardiomyopathy. We will, therefore, describe the condition simply as "noncompaction", retaining use of inverted commas in the subsequent text. The condition is usually diagnosed by indexing the thickness of the trabeculated layer of the left ventricular wall to that of the solid, or compacted, component. Different groups, nonetheless, have recommended various diagnostic criteria for establishing the presence of the phenotype. ${ }^{1-7}$ When present, the condition has been linked to heart failure, malignant arrhythmias, and thromboembolic events. It is currently considered by some to be a distinct entity. ${ }^{8}$ The number of publications relating to the condition, furthermore, has risen sharply in recent years. Thus, its citation has risen from relative obscurity to more than 1500 citations, with some 80 publications now emerging every year. This fact prompted Captur and colleagues to liken the alleged lesion to the $20^{\text {th }}$ century phenomenon of excessive diagnosis of mitral valvar prolapse. ${ }^{9}$ These considerations raise several questions. 
In the first place, we wonder whether the diagnosis of "noncompaction" will diminish in comparable fashion to the current diagnostic incidence of individuals with symptomatic prolapse of the leaflets of the mitral valve? It is already evident that a substantial proportion of those individuals who fulfil widely used criteria for the condition are asymptomatic and have good prognosis, providing evidence to support the notion that excessive trabeculations, in themselves, do not provide clinically useful information. ${ }^{10}$ This then raises the second question. Is the common presumption justified, as the term implies, that the presence of the excessive layer of trabecular myocardium in the postnatal heart is itself associated with inappropriate formation of the compact components of the ventricular walls? We can rephrase this question to ask if there is any evidence to show that the trabeculations, which unequivocally make up the greater thickness of the ventricular walls in the developing embryo, subsequently coalesce to form the compact component of the wall? If this is not the case, the third question is whether the presence of excessive trabeculation is no more than one end of the normal variation in persistence of the initial ventricular trabeculations? If the latter is the case, the fourth question is what else is required to bring some of these individuals to the attention of cardiologists? In this regard, there are then further areas of uncertainty. Is there an anatomical difference between individuals with increased trabeculations who do, or do not, have a reduced ejection fraction or a poor clinical outcome? And is the condition different in children as opposed to adults, and in those with otherwise normal as opposed to congenitally malformed hearts? In this review, representing as we do a group of individuals with varied interests and expertise, we dissect the evidence underscoring these various questions.

\section{Trabeculations in the animal kingdom}

The formation of the trabecular layer of the ventricular walls has recently been reviewed in the Canadian Journal of Cardiology. ${ }^{11}$ All vertebrate animals, during their embryogenesis, exhibit a highly trabeculated ventricle. ${ }^{12}$ The trabeculations within the embryonic ventricle are thin, less than 50 micrometres in diameter, so that they are adequately supplied by diffusion from the luminal blood in which they are bathed. As there is no coronary circulation during the early stages of embryogenesis, greater ventricular mass can be accomplished by growth of the trabeculations. ${ }^{13}$ This highly trabeculated and avascular ventricular design is maintained into adulthood in most of the cold-blooded vertebrate animals. ${ }^{14,15}$ In humans, in contrast, as is the case in warm-blooded vertebrate animals in general, the fetal period is characterized by a sustained growth of the compact wall, which makes up no more than the meagre outer shell of the embryonic ventricle. ${ }^{16-18}$

During fetal development, the thickness of the compact component of the wall outgrows the extent of the trabeculations many-fold. It is a fact that some of the embryonic-like trabeculations will either coalesce, or compact, or re-initiate growth, so as to assume a phenotype like that of the compact wall, with this process seen most clearly in the development of the papillary muscles. ${ }^{19}$ Eventually, trabeculations resembling those of the 
embryo are found only in the fine network that constitutes the Purkinje myocardium of the ventricular conduction system ${ }^{14,20}$ Because warm-blooded vertebrates have higher blood pressures than cold-blooded vertebrates, ${ }^{21}$ the developmental and evolutionary changes occurring during transformation from a trabeculated to compact wall could relate to forceproduction. The highly trabeculated ventricles of some species, such as the tuna fish, varanid lizards, python snakes, and crocodiles, all generate systemic blood pressures in the range of mammals. ${ }^{21}$ The stroke work done per gram ventricular tissue, irrespective of it being trabeculated or compact, is not different between cold- and warm-blooded vertebrates. ${ }^{22}$ And, in the most comprehensive review of the cardiac anatomy of mammals, the degree of trabeculation did not show any obvious correlation to the performance of the animal. ${ }^{23}$ Instead, the changes that take place during the transformation of a largely trabeculated to a predominantly compact wall may enable faster filling and emptying of the ventricles. It may thus be related to the high heart rates found in mammals and birds. ${ }^{24}$ When surveying the animal kingdom, therefore, we find no reason to believe that a trabeculated ventricle is at a mechanical disadvantage in terms of force-production. Indeed, we have established that the degree of trabeculation of the human heart does not predict

ejection fraction. ${ }^{10,12}$ In some individuals, nonetheless, the presence of excessive trabeculation is associated with reduced ejection fraction. It has yet to be established if there is a causal relationship between the two features.

\section{What is the experimental evidence supporting the notion of compaction of pre-existing trabeculations?}

Many of the current clinical descriptions relating to left ventricular "noncompaction", as indeed was the case in one of our original essays on the topic, introduce their work by presuming that the initial trabeculations observed in the developing human heart coalesce to form the compact components of the ventricular walls. The review provided by Sedmera and associates, which included one of the current authors, is usually cited as the basis for this presumption. ${ }^{25}$ The review, however, was just that. It was based primarily on interpretations of previous studies, although backed up by scanning electron microscopic studies of developing hearts, mostly from chicks, but also including examples of developing human hearts. No direct evidence was provided to show that the compact layers of the ventricular walls were formed from the initial trabeculations. If we now re-examine the morphologic evidence available from serially sectioned developing human hearts, we can see that the compact component of the ventricular walls is already well-formed at Carnegie stages 15 or 16 in the presence of a thick layer of persisting trabecular myocardium (Figure 1). Similar evidence is available from studies of developing murine hearts, with these developmental series showing that, although the papillary muscles of the mitral valve are unequivocally formed by compaction of pre-existing trabeculations, this is not necessarily the case for the compact component of the ventricular walls. Thus, lineage tracing of the embryonically formed trabeculations show that they contribute little, if anything, to the compact wall during the fetal period, when compaction is thought to occur (Figure 2). ${ }^{26}$ 
Evidence regarding the proliferation of the ventricular cardiomyocytes also favours formation of the compact component of the wall by thickening in its own right (Figure 3), concomitant with the establishment of vascular supply from the aorta, rather than by coalescence of pre-existing trabeculations. ${ }^{16-18}$ Thus, the initial embryonic trabeculations have an molecular identity distinct from that of the cardiomyocytes making up the eventual compact ventricular walls. It is only the cardiomyocytes of the His-Purkinje system that retain a phenotype like the embryonic trabeculations. ${ }^{27}$ The extent to which the initial embryonic trabeculations retain their identity, or change their identity, remains to be fully established.

\section{What is the evidence in human supporting the notion of compaction of pre-existing trabeculations?}

Should compaction of pre-existing trabeculations be an important contribution to formation of the compact wall, we would predict that, in the adult heart, the compact wall should be the greatest in the hearts with the least trabeculations. To test this possibility, we assessed the relation of the thicknesses of the compact and trabeculated layers of the left ventricular wall from our published data set of almost 3000 individuals. ${ }^{10}$ This analysis shows that the thickness of the compacted wall is positively related to trabecular thickness, rather than negatively related, as would be expected if compaction is an important process (Figure 4). At present, therefore, the evidence from experiments and human cohort studies suggest that the compact wall grows by intrinsic proliferation, rather than by compaction of the preexisting trabecular layer. If there is a contribution exclusively due to compaction of embryonic trabeculations, then this contribution is likely to be negligible. Furthermore, even if there is failure of compaction, this may not necessarily lead to a thinned compact component of the wall.

\section{What is the evidence relating to defects of proliferation?}

If not reflecting compaction of pre-existing trabeculations, it is certainly possible that "noncompaction" relates to defects of proliferation. ${ }^{28,29}$ At the time when compaction is thought to occur, which is around the transition from the embryonic to fetal period of development, the embryo weighs no more than 3 grams, with the heart itself weighing only 30 milligram. This is more than 4 orders of magnitude smaller than the adult arrangement. ${ }^{30}$ If "noncompaction" is found in an infant or adult, proliferation must by necessity have occurred by approximately normal rates, if not normal rates. Even if we accept the possibility that proliferation could be pathologically reduced, it could also be argued that the primary pathology is hypoplasia, irrespective of the extent of trabeculation. Thus, some estimates of ventricular mass made based on cardiac magnetic resonance imaging indicate that the left ventricular mass may be slightly smaller in people with better developed trabeculated layers. ${ }^{10,31}$ This would be consistent with a miniscule deficiency in proliferation. There is, however, considerable variation in the estimates made of the mass of the trabeculated component on the basis of magnetic resonance imaging. ${ }^{32} 33$ To the best of 
our knowledge, there has been no validation of how the mass of the trabeculated myocardial component, as estimated by echocardiography or magnetic resonance imaging, relates to overall left ventricular mass. The relatively small myocardial mass as noted in hearts with extensive trabeculations, therefore, may simply reflect the difficulty of accurately distinguishing trabeculations from the lumen of the intertrabecular recesses.

\section{What are the phenotypic features of "noncompaction"?}

A cursory examination of the images now readily available by searching the web for "noncompaction" reveals marked anatomical heterogeneity. Much of the variability reflects the different techniques used for the purposes of diagnosis. Further problems are inherent in the use of various criteria for diagnosis, since these vary for those employing echocardiography, as they do for those using the seemingly more accurate technique of magnetic resonance imaging. The commonest appearance now increasingly demonstrated in individuals undergoing assessment using magnetic resonance imaging is that of a left ventricular cavity with a luminal lace-like layer of trabeculations. This lace-like layer is discrete from the compact components of the ventricular walls, with the trabeculations themselves coalescing to become the papillary muscles of the mitral valve. Within such images, it is also frequent to observe the innermost parts of the luminal trabecular layer also to be relatively compacted. This produces a trilaminar rather than a bilaminar configuration. In this pattern, the compact wall forms a relatively thick layer, the lace-like trabeculations form the middle part of the wall, and the innermost trabeculations coalesce to form a thin layer, with this layer continuous basally with the papillary muscles of the mitral valve. The bases of the left ventricular papillary muscles, however, are known to be trabeculated in the healthy heart. ${ }^{34}$ It is possible, therefore, that the trilaminar mural architecture as seen in such examples of "noncompaction" is no more than an excessive representation of the normal setting. But these appearances are far from uniform.

\section{"Noncompaction" has a multiplicity of anatomical phenotypes}

It is, perhaps, surprising that, none of the images available from autopsied hearts replicate the trilaminar appearance described above. The images from autopsies typically, tend to reveal the presence of hypertrophied trabeculations, often in presence of a fibroelastotic endocardial surface layer. This produces a more obvious bilaminar arrangement. Some of the clinical images similarly show hypertrophied trabeculations, rather than the lace-like configuration visible in most of the magnetic resonance images. Most cartoons alleging to show the appearances of the lesion, in contrast, show deep recesses extending into the compact component of the wall. Such features are lacking in those cases diagnosed using magnetic resonance imaging. The images now available in these multiple publications suggest that there is no one arrangement that represents the condition, but rather a multiplicity of phenotypes. The distribution of the excessive trabeculations, furthermore, is also unhelpful. Petersen and colleagues ${ }^{7}$ found a very similar spread of trabeculations in healthy volunteers, athletes, patients with hypertrophic cardiomyopathy, hypertensive 
heart disease, dilated cardiomyopathy, aortic stenosis, and those with "noncompaction". This was subsequently confirmed by Jacquier and colleagues. ${ }^{4}$ Recent findings in large cohorts of healthy people now show that many individuals are positive for one criterion, or even several criteria, of "noncompaction" despite being asymptomatic. ${ }^{10} 31$ In one prospective study of 113 individuals positive for "noncompaction", the incidence of pathological events did not associate with the greatest extent of trabeculations. ${ }^{35}$ Instead, it was ventricular dilation, and fibrosis as assessed with late gadolinium enhancement, that achieved prognostic value. This, of course, is as found in other cardiomyopathies. A smaller study in children also revealed that excessive trabeculations did not have prognostic value. ${ }^{36}$ All of this evidence, therefore, supports the notion that presence of excessive trabeculation, in themselves, is an epiphenomenon. ${ }^{37}$

\section{What, then, are the genetics of excessive trabeculation?}

It is established that "noncompaction" often associates with mitochondrial disorders. ${ }^{38,39}$ Genetic defects are identified in some two-fifths of patients, and these defects are distributed on 20 different genes. ${ }^{40}$ The affected genes often code for proteins that constitute part of the contractile apparatus, or otherwise partake in the excitationcontraction coupling, such as the calcium handling ryanodine receptors. ${ }^{11,41,42}$ The same genes, however, are linked to cardiomyopathies in general. There is nothing, to the best of our knowledge, that points to the trabeculated myocardium being more affected than the compact myocardium. One exception in this regard may be $\mathrm{HCN} 4{ }^{43,44}$ The expression of the funny channel protein in the ventricles is known to be confined to the ventricular conduction system, which is itself derived from the embryonic trabeculations, but HCN4 is further expressed in the sinus and atrioventricular node. ${ }^{27,45}$ Thus, the clinical presentation of patients with mutations in HCN4 is confounded by the malfunctioning cardiac conduction system, again calling into question whether the excessive trabeculations are the causative agent, or simply an epiphenomenon. Perhaps the best attempt causally to relate genetic variation to "noncompaction" was made by Luxan and colleagues. ${ }^{46}$ In their study, all 10 carriers of mutations in the MIB1 gene of the two index families were positive for either the Petersen ${ }^{7}$ or Jacquier ${ }^{4}$ criterion for "noncompaction". Only 2 individuals, nonetheless, had heart failure. The remaining 8 carriers had benign "noncompaction, since they had normal left ventricular ejection fractions. ${ }^{47}$ More recently, Piccolo et al studied carriers of MIB2 mutation and only every second carrier satisfied the currently accepted criteria for "noncompaction" and all carriers had benign "noncompaction". ${ }^{48}$ As of yet, therefore, clinical genetics do not lend unequivocal support to the categorization of "noncompaction" as a distinct cardiomyopathy. Instead, some key findings can be considered in line with the notion of excessive trabeculation occurring as an epiphenomenon.

\section{Is there a difference between children and adults?}

Another of the key questions relates to whether the condition seen in children is different from adults. In case series of children diagnosed with "noncompaction", the prognosis 
appears to be poor, ${ }^{49}$ with some investigators finding a high mortality in individuals with low

ejection fractions. ${ }^{50}$ It is likely that those with more severe forms of excessive trabeculation present in childhood, ${ }^{47}$ perhaps explaining the more benign adult forms. Certainly, a survivor bias may partly explain the differences found. In neither childhood, nor in adult, studies of those with excessive trabeculations, however, is there convincing evidence that the presence or the degree of excessive trabeculation has an independent prognostic value after adjusting for common and known risk factors, such as left ventricular end-diastolic diameter or volume or ejection fraction. It remains an open question, furthermore, whether the extent of trabeculation in relation to the compact wall is constant from childhood to adulthood. In peripartum cardiomyopathy, for example, the trabeculated layer is known to thicken to a greater extent than the compact wall. ${ }^{51}$

\section{How best, then, can we describe the condition?}

Although the appearance is most frequently described as "noncompaction", we submit that all our discussion shows this to be less than satisfactory. The very use of the term implies that there has been some degree of failure of compaction during development. As shown above, there is no evidence of which we are aware to show that the initial embryonic trabeculations contribute substantially to the compact wall by a process of compaction. Extensive embryonic-like trabeculations can be expected if there is failed compaction, but, as one of us has recently shown in fetal cases that could be diagnosed as showing "noncompaction", the excessive trabeculations themselves do not have the embryonic identity of the initial trabecular layer. ${ }^{52}$ An alternative term, used as the condition began to achieve prominence, was "spongy myocardium". It is certainly the case that the lace-like configuration seen in many individuals has a sponge-like appearance, but the term is confounded by the inference that the "sponginess" is reminiscent of hearts found in coldblooded, or ectothermic, species. This association has been shown to be fals $\mathrm{e}^{12}$. Despite the frequent presumption that the trabecular layer is formed at the expense of the compact layer in "noncompaction", this is not the case. "Spongy" myocardium, therefore, is also less than ideal. "Hypertrabeculation" is another alternative that is again far from ideal. Does the prefix imply that there are too many trabeculations, or that the existing trabeculations are hypertrophied? And, if we are to judge the number of trabeculations, what is our point of reference? The term that, perhaps, best expresses the anatomical configuration underscoring the criteria proposed by Petersen and colleagues ${ }^{7}$ is excessive trabeculation. The extent of the trabeculations is certainly greater than that seen within the left ventricles of most normal individuals. Having described excessive trabeculation, it is then possible to explain whether the individual trabeculations are lace-like, or additionally hypertrophied and thickened, thus accounting for the variability to be found within published images.

\section{How excessive are the trabeculations?}


The burning current question is whether the excessive nature of the trabeculations represents an abnormality in itself, or is no more than an epiphenomenon. There is now much evidence accruing to favour the second alternative. ${ }^{39}$ The extensive investigation of the individuals collected together in the multi-ethnic study of atherosclerosis shows that there is a fairly normal distribution of the extent of trabeculation, forming a bell-shaped curve with a degree of rightward skewing (Figure 5). Those individuals making up the rightward end of the curve satisfy the accepted criteria established for diagnosis of "noncompaction" when using magnetic resonance imaging. Many individuals with excessive trabeculations, however, have none of the anticipated symptoms for "noncompaction". One-third of the cohort of patients with dilated cardiomyopathy studied by Amzulescu and colleagues, for example, also fulfilled these criteria. ${ }^{53}$ In commentating on their publication, one of us emphasised that "it seems that there is no convincing evidence to suggest that the degree of trabeculations has incremental prognostic value in asymptomatic low-risk populations and in dilated cardiomyopathy". ${ }^{54}$ Stöllberger and colleagues, furthermore, showed that the presence of excessive trabeculations did not predict clinical outcomes in adults. ${ }^{55}$ Further corroboration of these notions is now provided by the recent publications based, on the one hand, on a large cohort of healthy people, and on the other hand a prospective study of 113 individuals positive for "noncompaction". 31,35

\section{How, then, should we assess individuals with excessive trabeculation?}

There is significant controversy regarding the appropriate diagnostic criteria for "noncompaction". The current imaging techniques for the diagnosis have recently been reviewed, and controversies outlined. ${ }^{56}$ Some authors rely on a ratio of compacted to trabeculated myocardium of greater than 2 , or a trabecular mass of greater than $20 \%$ of total mass. ${ }^{4}$ The estimation of ventricular mass made by Jacquier et al, however, is greatly exaggerated, since they included the intertrabecular lumens as part of the trabeculated mass. Individuals show a wide spectrum in the extent and thickness of trabeculations. Many of these individuals have no evidence of heart disease, suggesting a potential diagnosis of "noncompaction" would be erroneous. We have shown in our discussion above that "noncompaction", as a term, probably does not reflect the underlying disease process. The use of the word "cardiomyopathy", furthermore, is problematic in the presence of a normally functioning heart. Some have suggested that, in addition to the presence of excessive trabeculations, various additional indices, such as mural thickness ${ }^{57}$ and ejection fraction, should be considered. The presence of a reduced ejection fraction, in the absence of another cause, certainly reflects a cardiomyopathic process, and may be an important marker for a disease process (Figure 6). Recently, Andreini and colleagues showed that dilation of the left ventricle, along with fibrosis as revealed by late gadolinium enhancement, do have prognostic value. ${ }^{35}$ This does suggest that a reduced ejection fraction, fibrosis in itself, or a dilated left ventricle, should be a prerequisite for diagnosing a clinically important condition. These parameters, nonetheless, are the hallmarks of cardiomyopathy in general. In this regard, Arbustini and her associates have suggested that, 
in many instances, "noncompaction" can adequately be described simply in terms of dilated, hypertrophic, restrictive cardiomyopathy, or arrhythmogenic right ventricular cardiomyopathy. ${ }^{39}$

\section{Is there a specific difference between "noncompaction" and dilated cardiomyopathy in the setting of excessive trabeculations?}

Whether or not "noncompaction" represents a distinct cardiomyopathy is also controversial. ${ }^{41} \mathrm{~A}$ thin compacted layer is seen by some as a prerequisite for the diagnosis. ${ }^{1,}$ 39, 41 In another study with age and sex matched adults, a maximal compacted thickness of less than $8 \mathrm{~mm}$ was proposed as a specific marker that would allow the differentiation of the pathological variant of excessive trabeculation, and thus prevent over-diagnosis. ${ }^{57}$ It is certainly possible that presence of excessive trabeculations, in some individuals, may simply be a marker for an unusually thin compact component of the ventricular wall, in other words an associated developmental abnormality, with more benign forms having a normal ejection fraction and wall thickness. ${ }^{47}$ In individuals with excessive trabeculations, it is those with lower ejection fraction, a higher end-diastolic volume, and heart failure symptoms who have increased mortality. ${ }^{58}$

We must ask, therefore, whether the presence of of a normal ejection fraction in the setting of excessive trabeculation excludes a disease process? Patients with excessive trabeculation and a normal ejection fraction do not appear to be at an increased risk of arrhythmias and embolic stroke. ${ }^{10}$ Of the dataset of more than 3000 asymptomatic participants making up the MESA cohort, the quintile with the greatest ratio between the thicknesses of the trabeculated and compact layers did not have greater risk for either arrhythmia or stroke. ${ }^{10}$ Excessive trabeculations, nonetheless, are known to be associated with several genetic abnormalities, ${ }^{39,59,60}$ including abnormalities in cardiomyocyte proteins, NOTCH signalling pathways, ${ }^{47}$ and calcium handling. ${ }^{42,61}$ These findings raise the possibility of a unifying developmental abnormality, which sometimes produces both an abnormal compacted layer and excessive trabeculations. The architecture of the trabecular layer, however, may also be relevant. Some individuals have the lace-like appearance, with thin trabeculations, while others have much thicker individual trabeculations. It remains uncertain as to whether these individuals have different clinical phenotypes and outcomes.

Case series have shown a high risk of ventricular arrhythmias, especially in symptomatic individuals, although more recent studies indicate a more benign outlook. ${ }^{47}$ The potential mechanism for arrhythmias in the setting of excessive trabeculation is currently unknown. ${ }^{47}$ Some individuals with ventricular arrhythmias in one study had decreased fractional shortening, and increased left ventricular end-diastolic dimensions, although those without arrhythmias had even lower measures. ${ }^{62}$ Worsening left ventricular ejection fraction is established as an important predictor for ventricular arrhythmias and mortality in children. ${ }^{50}$ Age at presentation, as well as the extent of the trabeculations, appears to be a major determinant of left ventricular ejection fraction. ${ }^{63}$ Ejection fraction, ${ }^{63,64}$ functional capacity ${ }^{63}$ 
or history of heart failure, ${ }^{65}$ sustained ventricular tachycardia ${ }^{65}$ and an enlarged left atrium ${ }^{65}$ have all been shown to predict mortality.

We suggest, therefore, that excessive trabeculation can occur in individuals with an excellent outlook, as an overlap syndrome with dilated cardiomyopathy secondary to a cardiomyocyte disease or related to thinner ventricular wall, ${ }^{66}$ or can appear secondary to a concurrent developmental abnormality (Figure 7). As already discussed, Arbustini and colleagues have proposed the sub-grouping of the finding of excessive trabeculation. They argued that individuals could be asymptomatic or symptomatic, with the symptoms reflecting associated cardiomyopathies, congenital malformations, being part of a syndrome, or acquired in relation to exercise or pregnancy. ${ }^{39}$ The suggested approach poses many questions, such as the prevalence, incidence, diagnostic criteria, prognosis and management of each of these suggested groupings, thus providing rich areas for future research.

\section{Conclusions}

There is growing evidence to indicate the lack of any connection between the mere presence of excessive ventricular trabeculation and mortality, the risk of arrhythmias, or stroke. Specifically, there is no convincing evidence that the extent of ventricular trabeculations has incremental prognostic value in asymptomatic individuals, those with a normal ejection fraction or even in those with dilated cardiomyopathy. "Noncompaction", as currently defined, is neither "arrested development", nor failure of the compaction of pre-existing trabeculations, nor a recapitulation of an evolutionary arrangement found in cold-blooded animals. Indeed, the condition, if it is one, is no more than excessive trabeculation! We conclude that compaction is not an important process for the formation of the compact wall, and that defining "noncompaction" without reference to left ventricular ejection fraction is unhelpful. We suggest that many individuals currently diagnosed as having "noncompaction" are fit and well, with an excellent prognosis. More research is needed to demonstrate if there is a clear phenotypic, pathophysiological, and prognostic difference between individuals with low ejection fraction with and without excessive trabeculations. Until then, it is questionable whether the entity, as currently identified, can be considered either as a separate disease, or as a useful diagnosis. We suggest that the term "noncompaction cardiomyopathy" should be abandoned, being replaced with a description of the trabeculations, along with risk factors such as ejection fraction (Figure 6).

\section{Figures}

Figure 1. Development of the compact and trabecular layers in humans

The images show "four chamber" sections through the heart of human embryos, from the sixth (Carnegie stage (CS) 12) through the seventh week (CS 17) of intrauterine 
development. They show the increasing thickness of the compact component of the wall after CS 15, with the trabeculations beginning to compact to form the papillary muscles of the mitral valve. There is no evidence, however, to show that compaction of the pre-existing trabecular layer forms the solid layer.

Figure 2. Lineage tracing of the embryonically formed trabeculations

Showing embryonically formed trabeculations, traced by their expression of Cx40 from 12.5, 14.5 and 16.5 days into development, have very little if any contribution to the compact wall. From Miquerol et al $2010^{26}$ reproduced with permission.

Figure 3 Development of the compact and trabecular layers in mice

The images, again in four chamber orientation, are from developing mouse hearts during the eleventh, twelfth, thirteenth, and fifteenth days of embryonic (E) development. The changes in thickness of the trabecular and compact layers parallel the changes seen in the developing human heart (Figure 1). As in the human heart, the trabeculations compact to form the papillary muscles (E14.5), but not the compact part of the ventricular wall.

Figure 4. Relationship between thickness of trabecular and compacted layers of myocardium in MESA cohort

In these patients with a normal ejection fraction, trabecular thickness and compacted wall thickness correlate suggesting that compaction is not an important process in formation of the compact ventricular wall.

Figure 5. Distortion of the frequency distribution of the ratio of trabeculation to compacted wall thickness by logarithmic transformation.

Showing the skewed distribution of the frequency of the ratio of trabeculation to compacted wall thickness and normal distribution after logarithmic transformation.

Figure 6. Proposed algorithm for excessive trabeculations management and risk assessment (adapted from Aung et al. ${ }^{67}$ )

A possible algorithm for the assessment of individuals with excessive trabeculation, which proposes that some individuals may not require long-term follow-up. Prognostic arrhythmias include non-sustained ventricular tachycardia (NSVT). LVEF, left ventricular ejection fraction; FH DCM/NCC, family history of dilated cardiomyopathy or non-compaction cardiomyopathy.

Figure 7. Possible categories with excessive trabeculations. 
Excessive trabeculations (defined as Petersen positive criteria ${ }^{7}$ ) with preserved ejection fraction (ETpEF). Excessive trabeculations with reduce ejection fraction (ETrEF) and dilated cardiomyopathy (DCM). Shown is a potential overlap syndrome with ETrEF and DCM and a distinct group (NCC) related to a developmental disorder as defined by Jenni and colleagues, where the diagnostic criteria include a thin compact layer. ${ }^{1}$

\section{References}

1. Jenni R, Oechslin E, Schneider J, Jost CA, Kaufmann P. Echocardiographic and pathoanatomical characteristics of isolated left ventricular non-compaction: a step towards classification as a distinct cardiomyopathy. Heart. 2001;86:666-671.

2. Chin TK, Perloff JK, Williams RG, Jue K, Mohrmann R. Isolated noncompaction of left ventricular myocardium. A study of eight cases. Circulation. 1990;82:507-513.

3. Stöllberger C, Finsterer J, Blazek G. Left ventricular hypertrabeculation/noncompaction and association with additional cardiac abnormalities and neuromuscular disorders. Am J Cardiol. 2002;90:899-902.

4. Jacquier A, Thuny F, Jop B, et al. Measurement of trabeculated left ventricular mass using cardiac magnetic resonance imaging in the diagnosis of left ventricular non-compaction. Eur. Heart. J. 2010;31:1098-1104.

5. Stacey RB, Andersen MM, St Clair M, Hundley WG, Thohan V. Comparison of systolic and diastolic criteria for isolated LV noncompaction in CMR. JACC Cardiovasc. Imaging. 2013;6:931-940.

6. Captur G, Muthurangu V, Cook C, et al. Quantification of left ventricular trabeculae using fractal analysis. J. Cardiovasc. Magn. Reson. 2013;15:36.

7. Petersen SE, Selvanayagam JB, Wiesmann F, et al. Left ventricular noncompaction: insights from cardiovascular magnetic resonance imaging. $J$. Am. Coll. Cardiol. 2005;46:101-105.

8. Oechslin EN, Attenhofer Jost CH, Rojas JR, Kaufmann PA, Jenni R. Long-term follow-up of 34 adults with isolated left ventricular noncompaction: a distinct cardiomyopathy with poor prognosis. J. Am. Coll. Cardiol. 2000;36:493-500.

9. Captur G, Flett AS, Jacoby DL, Moon JC. Left ventricular nonnoncompaction: the mitral valve prolapse of the 21 st century? Int. J. Cardiol. 2013;164:3-6.

10. Zemrak F, Ahlman MA, Captur G, et al. The relationship of left ventricular trabeculation to ventricular function and structure over a 9.5- 
year follow-up: the MESA study. J. Am. Coll. Cardiol. 2014;64:19711980.

11. Captur G, Syrris P, Obianyo C, Limongelli G, Moon JC. Formation and Malformation of Cardiac Trabeculae: Biological Basis, Clinical Significance, and Special Yield of Magnetic Resonance Imaging in Assessment. Can. J. Cardiol. 2015;31:1325-1337.

12. Jensen B, Agger P, de Boer BA, et al. The hypertrabeculated (noncompacted) left ventricle is different from the ventricle of embryos and ectothermic vertebrates. Biochimica et Biophysica Acta (BBA)Molecular Cell Research. 2016;1863:1696-1706.

13. Sedmera D. Function and form in the developing cardiovascular system. Cardiovasc. Res. 2011;91:252-259.

14. Jensen B, Boukens BJ, Postma AV, et al. Identifying the evolutionary building blocks of the cardiac conduction system. PLoS One. 2012;7:e44231.

15. Farrell A, Farrell N, Jourdan H, Cox G. A perspective on the evolution of the coronary circulation in fishes and the transition to terrestrial life. Ontogeny and Phylogeny of the Vertebrate Heart: Springer; 2012:75-102.

16. Sedmera D, Reckova M, DeAlmeida A, et al. Spatiotemporal pattern of commitment to slowed proliferation in the embryonic mouse heart indicates progressive differentiation of the cardiac conduction system. Anat. Rec. A Discov. Mol. Cell. Evol. Biol. 2003;274:773-777.

17. de Boer BA, van den Berg G, de Boer PA, Moorman AF, Ruijter JM. Growth of the developing mouse heart: an interactive qualitative and quantitative 3D atlas. Dev. Biol. 2012;368:203-213.

18. Sizarov A, Ya J, de Boer BA, Lamers WH, Christoffels VM, Moorman AF. Formation of the building plan of the human heart: morphogenesis, growth, and differentiation. Circulation. 2011;123:1125-1135.

19. Anderson RH. The relationship between the positions of the left ventricular papillary muscles and the direction of the QRS axis. $J$. Electrocardiol. 2016;49:675-679.

20. Sizarov A, Devalla HD, Anderson RH, Passier R, Christoffels VM, Moorman AF. Molecular analysis of patterning of conduction tissues in the developing human heart. Circulation. Arrhythmia and electrophysiology. 2011;4:532-542.

21. Jensen B, Wang T, Christoffels VM, Moorman AF. Evolution and development of the building plan of the vertebrate heart. Biochim. Biophys. Acta. 2013;1833:783-794.

22. Hillman SS, Hedrick MS. A meta-analysis of in vivo vertebrate cardiac performance: implications for cardiovascular support in the evolution of endothermy. J. Exp. Biol. 2015;218:1143-1150.

23. Rowlatt U. Comparative anatomy of the heart of mammals. Zool. J. Linn. Soc. 1990;98:73-110. 
24. Burggren WW, Christoffels VM, Crossley DA, 2nd, et al. Comparative cardiovascular physiology: future trends, opportunities and challenges. Acta physiologica. 2014;210:257-276.

25. Sedmera D, Pexieder T, Vuillemin M, Thompson RP, Anderson RH. Developmental patterning of the myocardium. Anat. Rec. 2000;258:319337.

26. Miquerol L, Moreno-Rascon N, Beyer S, et al. Biphasic development of the mammalian ventricular conduction system. Circ. Res. 2010;107:153161.

27. van Weerd JH, Christoffels VM. The formation and function of the cardiac conduction system. Development. 2016;143:197-210.

28. Arndt A-K, Schafer S, Drenckhahn J-D, et al. Fine mapping of the 1p36 deletion syndrome identifies mutation of PRDM16 as a cause of cardiomyopathy. The American Journal of Human Genetics. 2013;93:6777.

29. Kodo K, Ong SG, Jahanbani F, et al. iPSC-derived cardiomyocytes reveal abnormal TGF-beta signalling in left ventricular non-compaction cardiomyopathy. Nat. Cell Biol. 2016;18:1031-1042.

30. de Bakker BS, de Jong KH, Hagoort J, et al. An interactive threedimensional digital atlas and quantitative database of human development. Science. 2016;354:aag0053.

31. Weir-McCall JR, Yeap PM, Papagiorcopulo C, et al. Left Ventricular Noncompaction: Anatomical Phenotype or Distinct Cardiomyopathy? J. Am. Coll. Cardiol. 2016;68:2157-2165.

32. Bricq S, Frandon J, Bernard M, et al. Semiautomatic detection of myocardial contours in order to investigate normal values of the left ventricular trabeculated mass using MRI. J. Magn. Reson. Imaging. 2015.

33. Choudhary P, Hsu CJ, Grieve S, et al. Improving the diagnosis of LV non-compaction with cardiac magnetic resonance imaging. Int. J. Cardiol. 2015;181:430-436.

34. Axel L. Papillary muscles do not attach directly to the solid heart wall. Circulation. 2004;109:3145-3148.

35. Andreini D, Pontone G, Bogaert J, et al. Long-term prognostic value of cardiac magnetic resonance in left ventricle noncompaction: a prospective multicenter study. J. Am. Coll. Cardiol. 2016;68:2166-2181.

36. Zuckerman WA, Richmond ME, Singh RK, Carroll SJ, Starc TJ, Addonizio LJ. Left-ventricular noncompaction in a pediatric population: predictors of survival. Pediatr. Cardiol. 2011;32:406-412.

37. Aung N, Zemrak F, Petersen SE. Left Ventricular Noncompaction, or Is It? J. Am. Coll. Cardiol. 2016;68:2182-2184.

38. Finsterer J. Cardiogenetics, neurogenetics, and pathogenetics of left ventricular hypertrabeculation/noncompaction. Pediatr. Cardiol. 2009;30:659-681. 
39. Arbustini E, Favalli V, Narula N, Serio A, Grasso M. Left Ventricular Noncompaction: A Distinct Genetic Cardiomyopathy? J. Am. Coll. Cardiol. 2016;68:949-966.

40. Hoedemaekers YM, Klaassen S. Left Ventricular Noncompaction. Clinical Cardiogenetics: Springer; 2016:113-135.

41. Arbustini E, Weidemann F, Hall JL. Left ventricular noncompaction: a distinct cardiomyopathy or a trait shared by different cardiac diseases? $J$. Am. Coll. Cardiol. 2014;64:1840-1850.

42. Wilsbacher L, McNally EM. Genetics of Cardiac Developmental Disorders: Cardiomyocyte Proliferation and Growth and Relevance to Heart Failure. Annu. Rev. Pathol. 2016;11:395-419.

43. Milano A, Vermeer AM, Lodder EM, et al. HCN4 mutations in multiple families with bradycardia and left ventricular noncompaction cardiomyopathy. J. Am. Coll. Cardiol. 2014;64:745-756.

44. Schweizer PA, Schröter J, Greiner S, et al. The symptom complex of familial sinus node dysfunction and myocardial noncompaction is associated with mutations in the HCN4 channel. J. Am. Coll. Cardiol. 2014;64:757-767.

45. Liang $\mathrm{X}$, Wang G, Lin L, et al. HCN4 dynamically marks the first heart field and conduction system precursors. Circ. Res. 2013;113:399-407.

46. Luxán G, Casanova JC, Martinez-Poveda B, et al. Mutations in the NOTCH pathway regulator MIB1 cause left ventricular noncompaction cardiomyopathy. Nat. Med. 2013;19:193-201.

47. Towbin JA, Lorts A, Jefferies JL. Left ventricular non-compaction cardiomyopathy. Lancet. 2015;386:813-825.

48. Piccolo P, Attanasio S, Secco I, et al. MIB2 variants altering NOTCH signalling result in left ventricle hypertrabeculation/non-compaction and are associated with Menetrier-like gastropathy. Hum. Mol. Genet. 2016:ddw365.

49. Jefferies JL, Wilkinson JD, Sleeper LA, et al. Cardiomyopathy Phenotypes and Outcomes for Children With Left Ventricular Myocardial Noncompaction: Results From the Pediatric Cardiomyopathy Registry. $J$. Card. Fail. 2015;21:877-884.

50. Brescia ST, Rossano JW, Pignatelli R, et al. Mortality and sudden death in pediatric left ventricular noncompaction in a tertiary referral center. Circulation. 2013;127:2202-2208.

51. Gati S, Papadakis M, Papamichael N, et al. Reversible de novo left ventricular trabeculations in in pregnant women: implications for the diagnosis of left ventriculaer non-compaction cardiomyopathy in low risk poplations. Circulation. 2014.

52. Jensen B, van der Wal AC, Moorman AF, Christoffels VM. Excessive trabeculations in noncompaction do not have the embryonic identity. Int. J. Cardiol. 2016;In press:10.1016/j.ijcard.2016.11.089. 
53. Amzulescu MS, Rousseau MF, Ahn SA, et al. Prognostic Impact of Hypertrabeculation and Noncompaction Phenotype in Dilated Cardiomyopathy: A CMR Study. JACC Cardiovasc. Imaging. 2015;8:934-946.

54. Petersen SE. Left Ventricular Noncompaction: A Clinically Useful Diagnostic Label? JACC Cardiovasc. Imaging. 2015;8:947-948.

55. Stöllberger C, Blazek G, Gessner M, Bichler K, Wegner C, Finsterer J. Age-dependency of cardiac and neuromuscular findings in adults with left ventricular hypertrabeculation/noncompaction. Am J Cardiol. 2015;115:1287-1292.

56. Chebrolu LH, Mehta AM, Nanda NC. Noncompaction cardiomyopathy: The role of advanced multimodality imaging techniques in diagnosis and assessment. Echocardiography. 2017.

57. Gebhard C, Stahli BE, Greutmann M, Biaggi P, Jenni R, Tanner FC. Reduced left ventricular compacta thickness: a novel echocardiographic criterion for non-compaction cardiomyopathy. J. Am. Soc. Echocardiogr. 2012;25:1050-1057.

58. Greutmann M, Mah ML, Silversides CK, et al. Predictors of adverse outcome in adolescents and adults with isolated left ventricular noncompaction. Am J Cardiol. 2012;109:276-281.

59. Thevathasan W, Squier W, MacIver DH, Hilton DA, Fathers E, HiltonJones D. Oculopharyngodistal myopathy-A possible association with cardiomyopathy. Neuromuscul. Disord. 2011;21:121-125.

60. Finsterer J, Stollberger C, Towbin JA. Left ventricular noncompaction cardiomyopathy: cardiac, neuromuscular, and genetic factors. Nat. Rev. Cardiol. 2017.

61. Klaassen S, Probst S, Oechslin E, et al. Mutations in sarcomere protein genes in left ventricular noncompaction. Circulation. 2008;117:28932901.

62. Caliskan $\mathrm{K}$, Ujvari B, Bauernfeind $\mathrm{T}$, et al. The prevalence of early repolarization in patients with noncompaction cardiomyopathy presenting with malignant ventricular arrhythmias. J. Cardiovasc. Electrophysiol. 2012;23:938-944.

63. Aras D, Tufekcioglu O, Ergun K, et al. Clinical features of isolated ventricular noncompaction in adults long-term clinical course, echocardiographic properties, and predictors of left ventricular failure. $J$. Card. Fail. 2006;12:726-733.

64. Stanton $\mathrm{C}$, Bruce $\mathrm{C}$, Connolly $\mathrm{H}$, et al. Isolated left ventricular noncompaction syndrome. Am J Cardiol. 2009;104:1135-1138.

65. Lofiego C, Biagini E, Pasquale F, et al. Wide spectrum of presentation and variable outcomes of isolated left ventricular non-compaction. Heart. 2007;93:65-71. 
66. MacIver DH. A new understanding and definition of non-compaction cardiomyopathy using analysis of left ventricular wall mechanics and stresses. Int. J. Cardiol. 2014;174:819-821.

67. Aung N, Zemrak F, Mohiddin SA, Petersen SE. LV Noncompaction Cardiomyopathy or Just a Lot of Trabeculations? JACC Cardiovasc. Imaging. 2016; In press. 


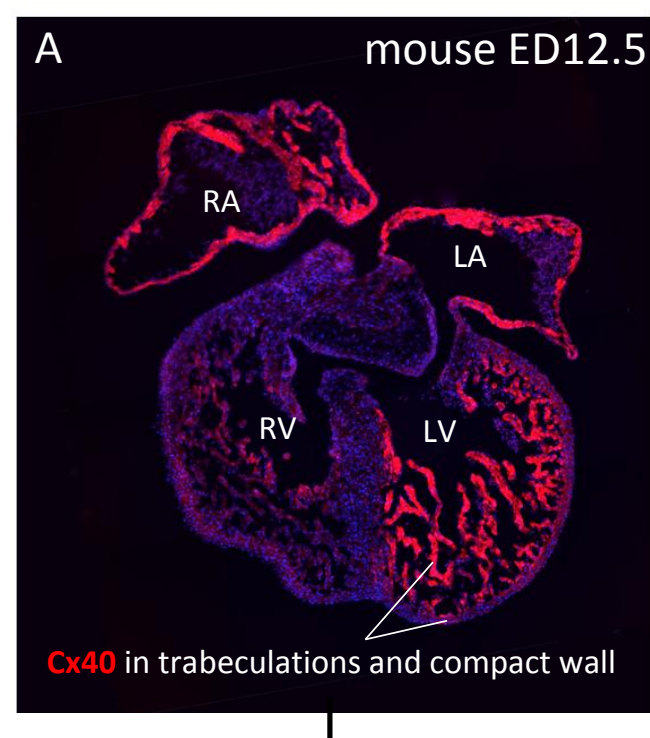

Cx40 lineage traced from ED12.5 to adult

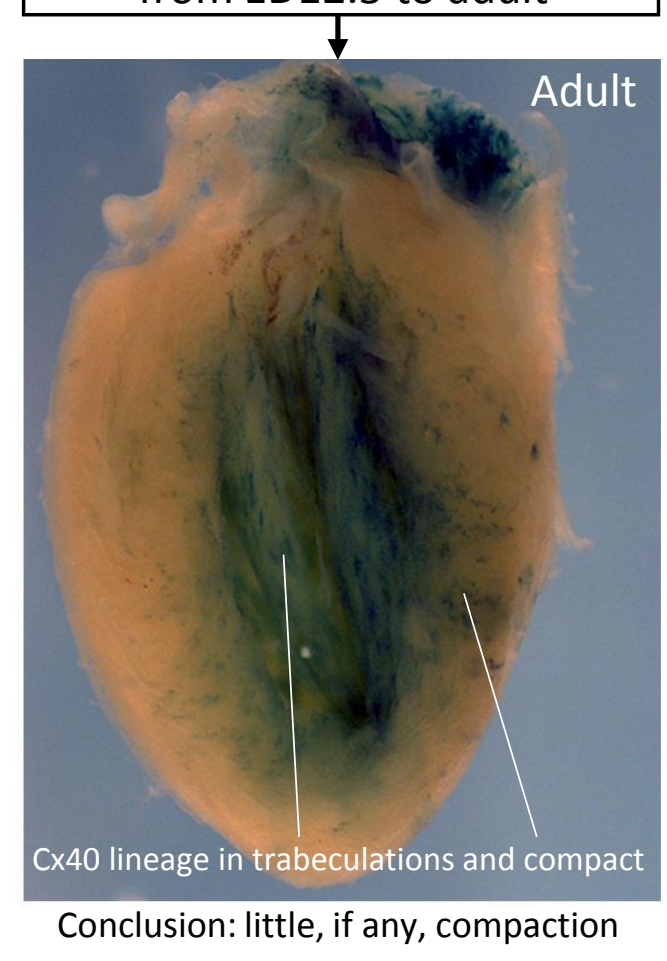

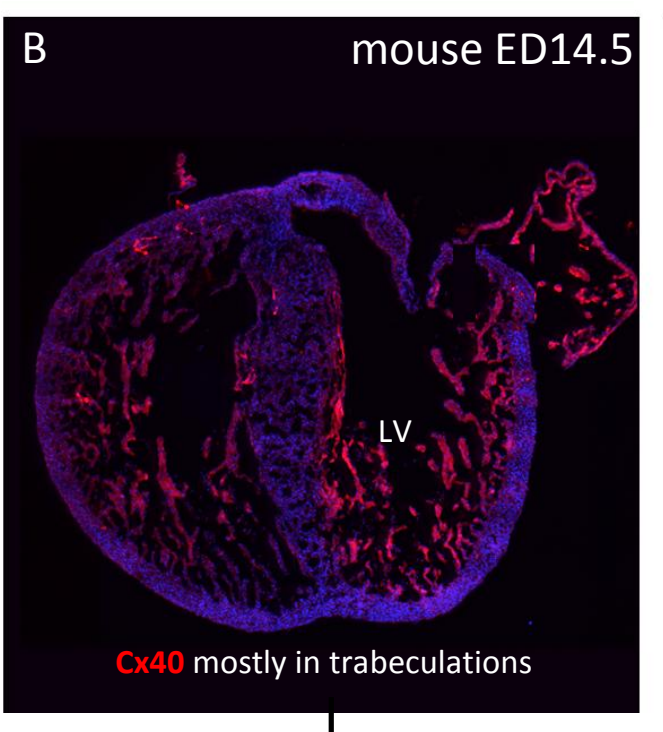

Cx40 lineage traced from ED14.5 to adult

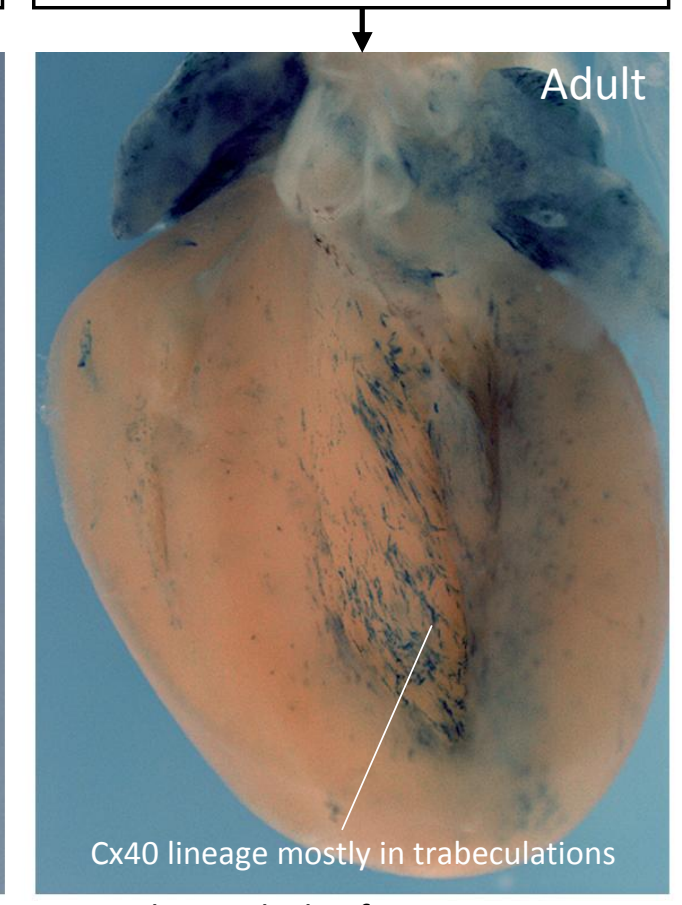

Conclusion: little, if any, compaction

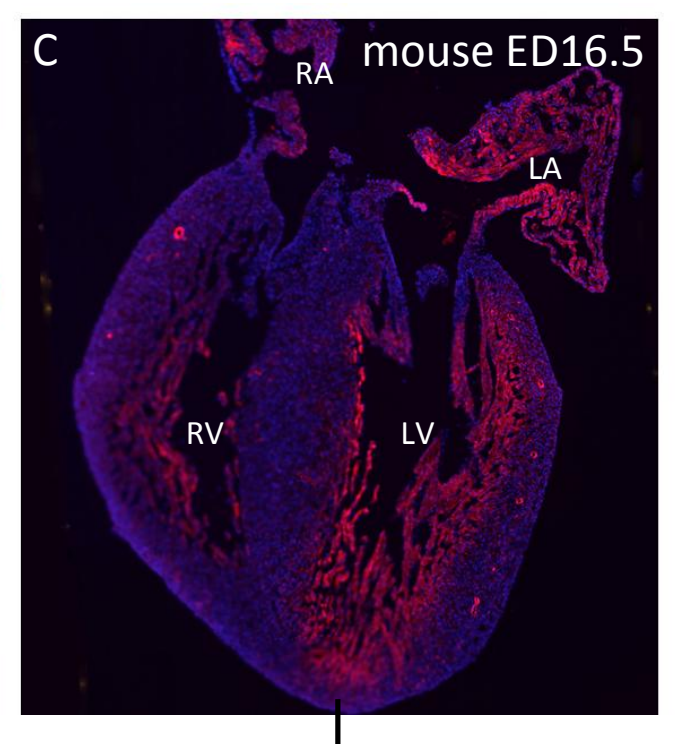

Cx40 lineage traced from ED16.5 to adult

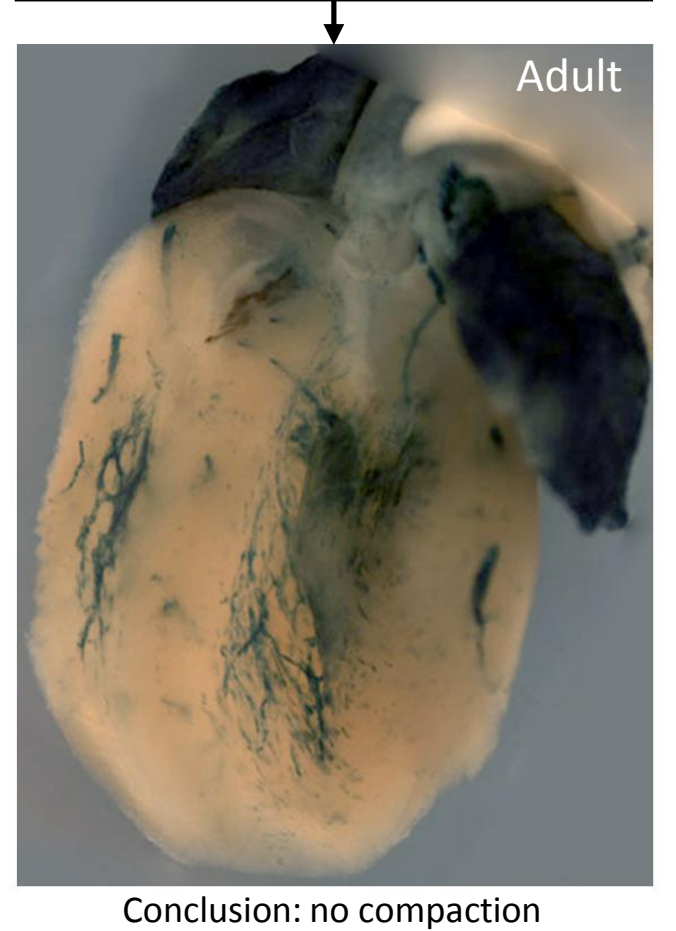

\section{Figure 2}




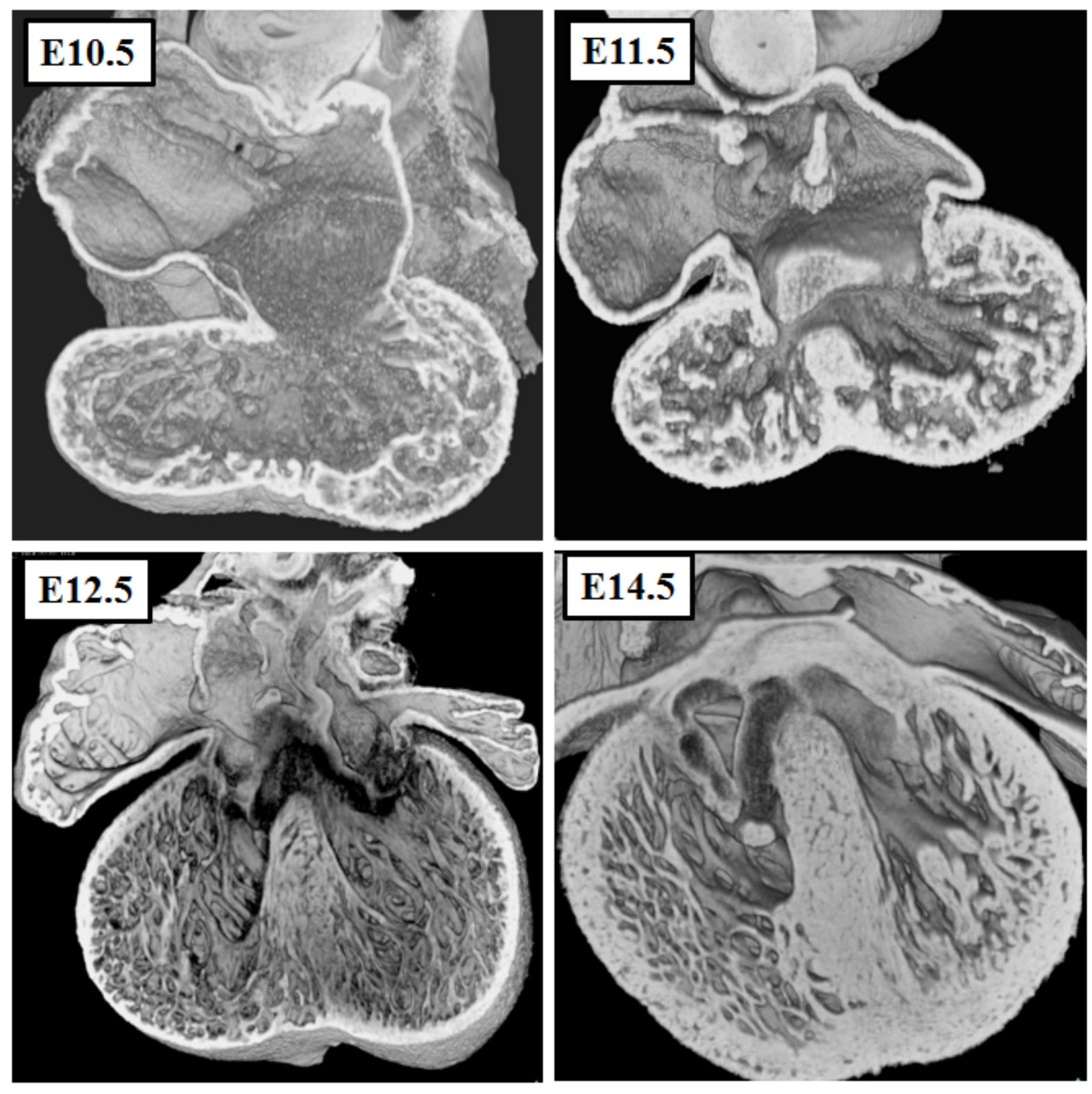

Figure 3 


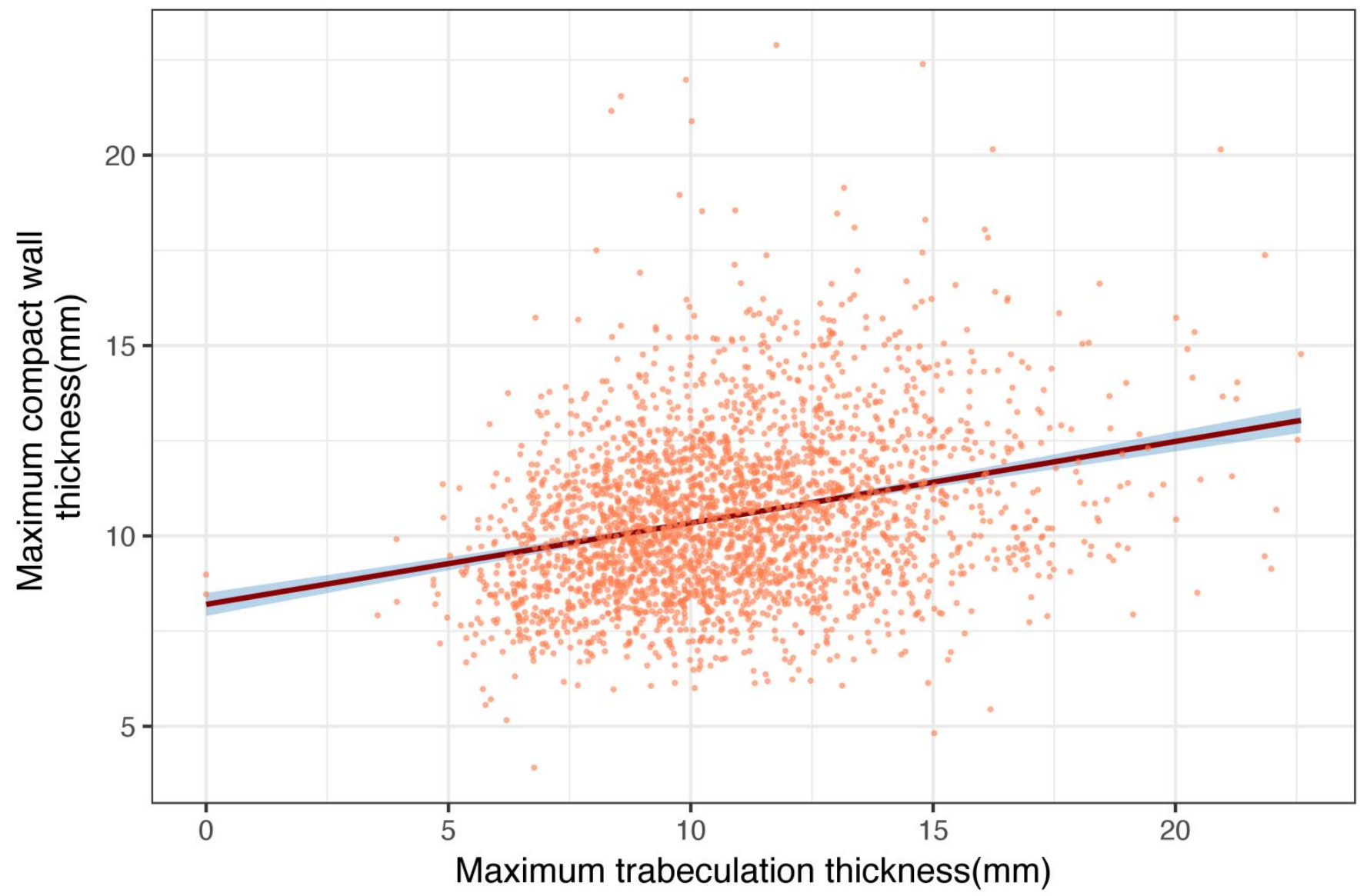

\section{Figure 4}




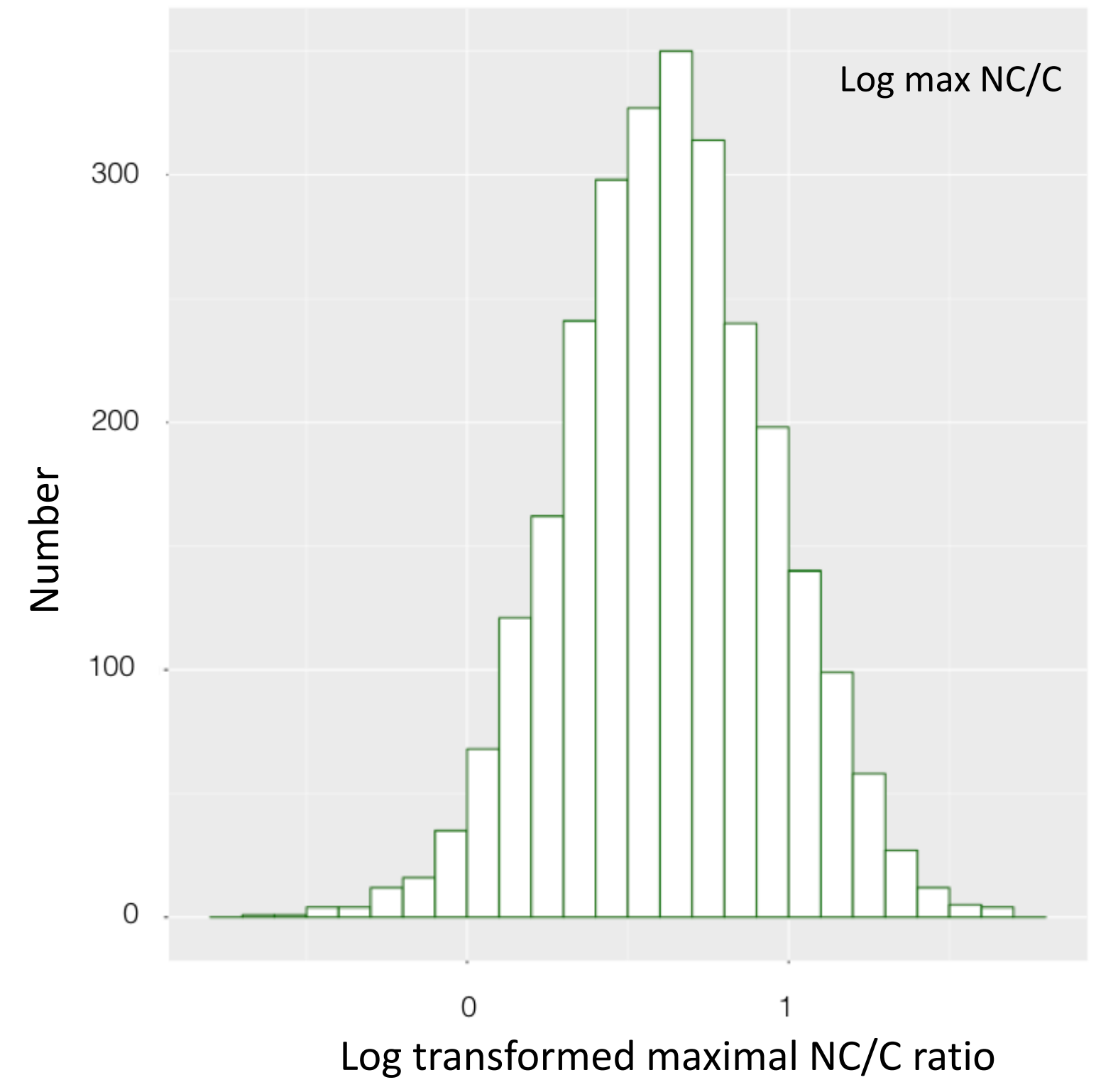

Figure 5 


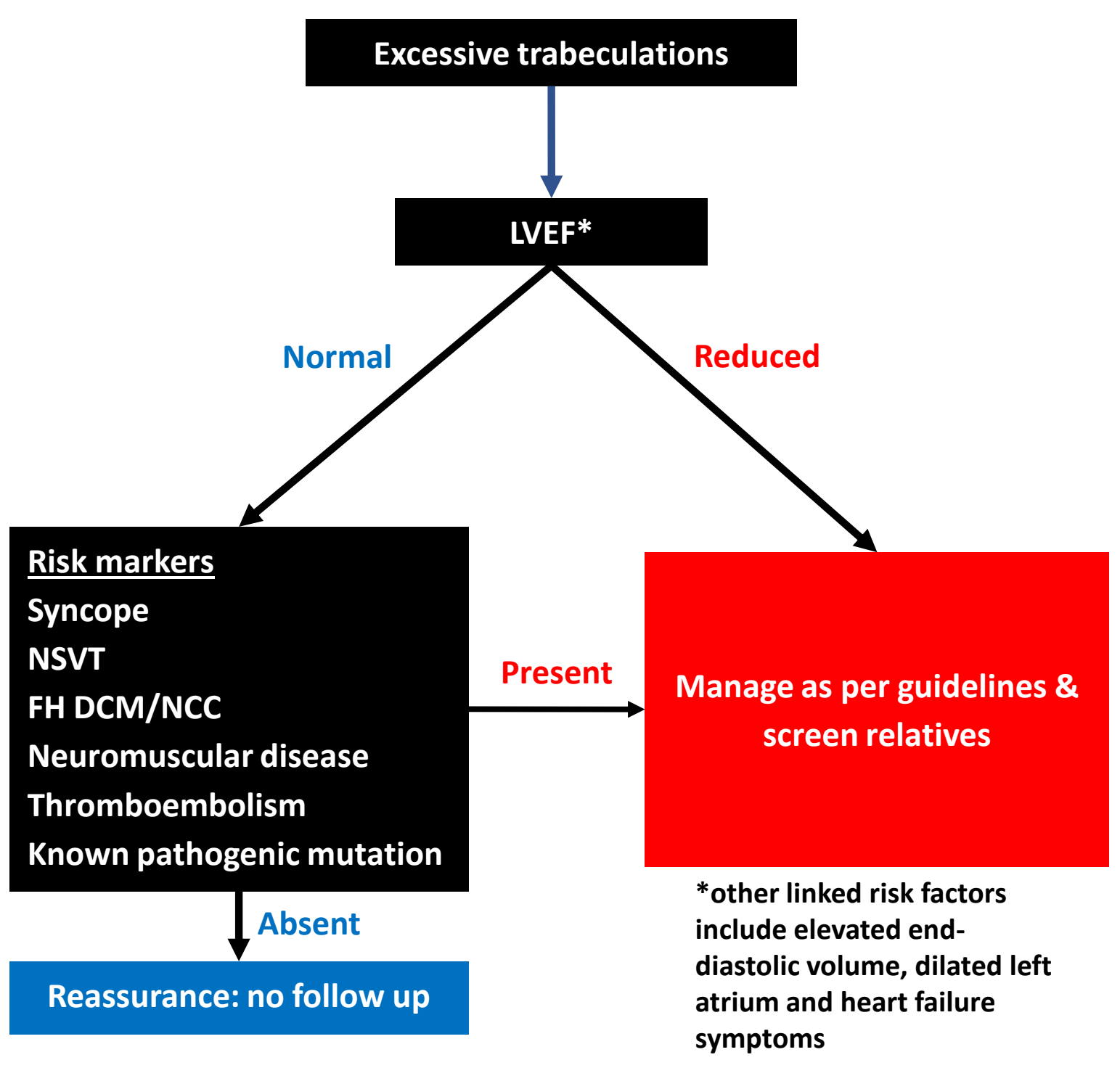




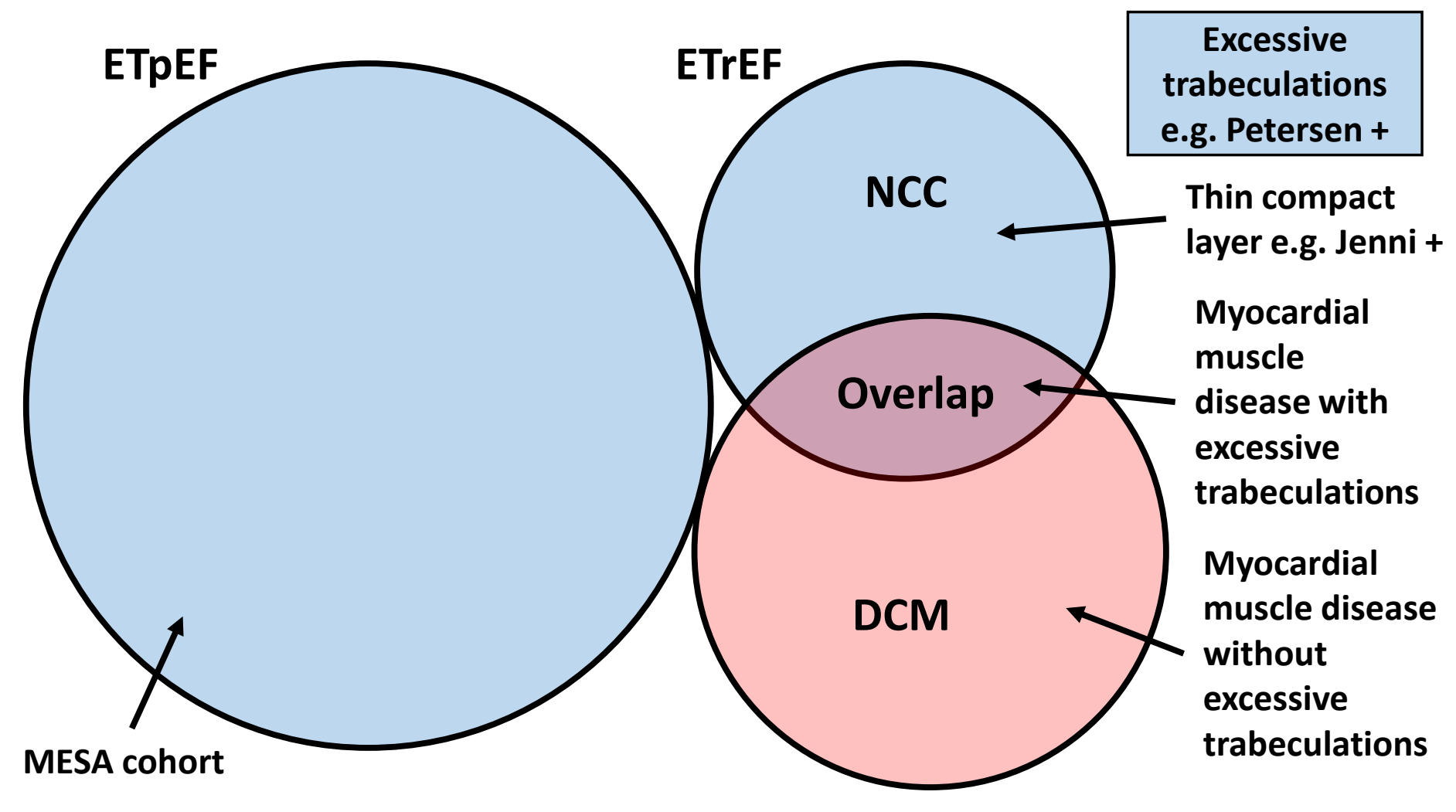

\section{Figure 7}


Supplementary Material
Click here to download Supplementary Material: RightsLink Printable License For Figure 2.pdf

Supplementary Material
Click here to download Supplementary Material: RightsLink Printable License For Figure 2.pdf

che tar (a)

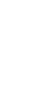

(n)

